\title{
Comparison of low-dose contrast computed tomography angiography findings with surgical results in living kidney donors
}

\author{
๑Hasan Gündoğdu¹, @Sibel Aydın Aksu², V. Melih Kara ${ }^{3}$ \\ ${ }^{1}$ Recep Tayyip Erdogan University Faculty of Medicine, Department of Radiology, Rize, Turkey \\ ${ }^{2}$ Health Sciences University, Haydarpaşa Numune Health Practice Center, Radiology Clinic, İstanbul, Turkey \\ ${ }^{3}$ Başakşehir Çam and Sakura City Hospital, Department of General Surgery, İstanbul, Turkey
}

Cite this article as: Gündoğdu H, Aydın Aksu S, Kara VM. Comparison of low-dose contrast computed tomography angiography findings with surgical results in living kidney donors. J Health Sci Med 2022; 5(1): 161-166.

\begin{abstract}
Aim: To analyze the image quality and diagnostic performance of CT angiography using low dose $(60 \mathrm{ml})$ contrast medium for living kidney donors and compare with surgical results.

Material and Method: Angiographic findings of 81 renal donor Candidates in 128-slice MDCT were evaluated by two independent radiologists in terms of renal artery number, early bifurcation, renal vein variations, pelvicalyceal system and ureter variations. Results were compared with intraoperative findings. The image quality, diagnostic performance and interobserver agreement of MDCT obtained with low dose contrast material were analyzed.

Results: The mean age of the 81 living kidney donors included in the study was 49 \pm 12 (24-68) years. Left nephrectomy was performed in $71 \%(n=64)$ and right nephrectomy in $29 \%(n=17)$ of the donors. Intraoperative accessory arteries were detected in $22.2 \%(\mathrm{n}: 18)$ of the donors. The specificity, sensitivity, and accuracy for detecting accessory artery variation in MDCT were $100 \%, 88.9 \%$, and $97.5 \%$, respectively. Early bifurcation was observed in $21 \%(n=17)$ of the donors. Specificity, sensitivity and accuracy for early bifurcation detection were $98.4 \%, 94.1 \%$ and $97.5 \%$, respectively. Renal vein variation was detected in $12.3 \%(\mathrm{n}=10)$ of the donors. Specificity, sensitivity, and accuracy for renal vein variation detection were $100 \%$. Variations of the pelvicalyceal system and ureter were observed in $3.7 \%(n=3)$ of the donors. The specificity, sensitivity, and accuracy for detecting pelvicalyceal system and ureteral variations were $100 \%$. Interobserver agreement was excellent in detecting variations of accessory arteries, renal venous anomalies, pelvicalyceal system and ureters by MDCT (kappa: 1,000; $\mathrm{p}<0.001$ ). It was higher in early bifurcation detection (kappa: $0.853 ; \mathrm{p}<0.001$ ).

Conclusion: MDCT angiography with a lower dose of iodine contrast at $60 \mathrm{~mL}$ in kidney donors is sufficient to detect vascular anomalies and provide anatomical information. It is possible to reduce the contrast agent dose in CTA without affecting the preoperative evaluation.
\end{abstract}

Keywords: Multidetector computed tomography, kidney, transplantation, contrast dose, contrast dose reduction

\section{INTRODUCTION}

Transplantation from a living donor is the most effective treatment option in patients with end-stage renal disease (1). In transplantation operations performed from living donors, preoperative evaluation and removal of the organ to be transplanted in a way that causes minimal damage to the donor and the organ itself is of great importance (2).

Clinical evaluation, laboratory tests and a comprehensive radiological study are necessary to determine the quality of the graft organ, to prevent future problems in the donor, to increase the success of the operation and to ensure that the graft works well in the recipient $(3,4)$.
Detailed pre-operative knowledge of the donor's renal anatomy helps to minimize the risks of intraoperative bleeding, vascular or ureteral injury $(5,6)$.

In the past, living kidney donors were subjected to preoperative evaluation with excretory urography and renal catheter angiography. However, because angiography is an invasive procedure and its value is limited in the detailed evaluation of renal venous anomalies, cross-sectional examination methods have taken its place (7). 
The vascular and pelvicalyceal anatomy of the donor can be identified by CT or MRI (8).

With the introduction of multi detector computed tomography (MDCT), increased scanning speed, higher spatial resolution, thinner sections and superior image quality have been achieved. CT has become a safe and widely accepted method for the preoperative evaluation of the renal vasculature, due to the higher spatial resolution, acquisition rate, and greater ability to detect small accessory arteries than MRI $(9,10)$.

The use of high-dose contrast media in computed tomography can cause acute kidney injury (CI-AKI), known as contrast-induced nephropathy not associated with another nephrotoxic event, when there is a sudden increase in serum creatinine after exposure to iodinated contrast media. This condition is normally self-limiting, but can lead to increased morbidity and mortality $(11,12)$.

The degree of renal artery opacification defines the technical adequacy of CT Angiography (CTA) scanning and is proportional to the rate and dose of contrast agent administration. Recent advances in CT technology and faster image acquisition times can shorten the time required for opacification of the vascular system. Therefore, it makes it possible to reduce the contrast agent dose used in CTA (13).

Due to the difficulty of finding renal donors, the contrast agent dose to be applied to the kidney to be transplanted is important. It has been reported in the literature that $75-120 \mathrm{ml}$ of contrast material is used in preoperative CT angiography in renal donors (14-16).

The use of less contrast reduces the risk of potential complications associated with an iodinated contrast agent, such as an allergic reaction or nephrotoxicity. Therefore, the contrast dose should be reduced to the lowest dose that will not affect the imaging quality (13).

The aim of this study is to analyze the image quality and diagnostic performance of CTA using $60 \mathrm{ml}$ of contrast medium for living kidney donors and compare it with surgical results.

\section{MATERIAL AND METHOD}

The study was reviewed and applied by Non-Interventional Clinical Researchs Ethics Committee of Health Sciences University, Haydarpaşa Numune Training and Research Hospital Health Application Center (Date: 02.08.2021, Decision No: 2021/197-3396). All procedures were carried out in accordance with ethical rules and the principles of the Declaration of Helsinki.

Between February 2015 and April 2021, 110 potential living donors who underwent CTA imaging before renal transplantation in our hospital were retrospectively reviewed. Nineteen patients were excluded from the study because nephrectomy was not performed due to malignancy and/or positive cross-match results, and 10 patients were excluded because the contrast medium rate $(3.5 \mathrm{ml} / \mathrm{sec})$ was different. 81 patients were included in the study.

CTA imaging in renal donors was performed with 128-slice CT (Optima CT 660, Logic Healthcare, Waukesha, USA) with $120 \mathrm{Kv}$ radiation dose, $150 \mathrm{~mA}$, tube output between 200-400, 0.6 pitch, 0.4 sec rotation time, $0.625 \mathrm{~mm}$ slice thickness and It was done with 40 mm collimation.

The patients were taken to the to the CT scan as fasting for at least 6 hours and drinking 250-500 cc of water in the last half hour. The CTA procedure and breathing instructions were explained to each patient.

$60 \mathrm{ml}$ of contrast material (iohexol, Omnipaque 350, Amersham Health, Princeton, NJ) was given to the patients through an $18 \mathrm{G}$ cannula with an automatic injector (Ulrich Inject CT Motion, Ulrich Medical, Buchbrunnenweg, Germany) at a rate of $4 \mathrm{ml} / \mathrm{sec}$. After the contrast application, $40 \mathrm{cc}$ of saline was administered at the same rate.

Imaging was performed by taking arterial $(25 \mathrm{sec})$, portal $(60 \mathrm{sec})$ and late venous $(300 \mathrm{sec})$ phases in the craniocaudal direction. Scanning area starting from the top of the diaphragm domes; It was determined to be just below the common iliac arteries in the arterial and venous phase, and up to the symphysis pubis in the late venous phase.

All acquired images were transferred to the workstation (Advanced Workstation, GE Healthcare, Milwaukee, WI, USA) for postprocessing.

Two experienced independent radiologists (S.A and H.G.) reviewed images from each CT scan at the workstation preoperatively. Image analyzes were performed without knowledge of other reviewers or surgical results.

Multiplane remodeling (MPR) and volume rendering (VR) techniques were generally used for 3D CT angiography. However, the maximum intensity projection (MIP) technique was used especially when needed for small vessels.

Renal artery anatomy was evaluated in arterial phase, venous anatomy was evaluated in arterial and/or portal phase, and collecting system anatomy and variations were evaluated in late venous phase.

Both radiologists recorded the number of renal arteries. Any branch within $2.0 \mathrm{~cm}$ of the aorta was classified as 
early branching. Other associated findings, including stenosis and aneurysms, were recorded for each artery. Renal vein anatomy was evaluated for the presence of retroaortic and circumaortic veins. Pelvicalyceal system and ureteral anomalies were recorded.

None of the patients developed complications related to contrast material. In addition, there was no patient who could not be evaluated due to poor image quality or insufficiency.

Donor nephrectomy was performed between 4 weeks and 6 months (mean 3 months) after CT examination. Transplant surgeons recorded the side of the nephrectomy kidney, the number of renal arteries, the presence of early branching arteries, the presence of renal vein anomalies, pelvicalyceal system and ureter anomalies.

\section{Statistical Analysis}

Statistical analyzes were performed with IBM SPSS Statistics, Version 23.0 (SPSS Inc., Chicago, USA) and MedCalc ${ }^{\circledast}$ Statistical Software version 20 (MedCalc Software Ltd, Ostend, Belgium; https://www.medcalc. org; 2021). Categorical data of both groups were reported as frequencies and percentages within the groups $(n, \%)$. The relationship between observers and surgery was assessed by kappa concordance analyses. In addition, the diagnostic performance of the observers according to surgery was evaluated and reported as \% sensitivity and \% specificity. The limit of significance was accepted as $\mathrm{p}<0.05$.

\section{RESULTS}

The mean age of 81 living kidney donors included in the study was $49 \pm 12$ (min-max:24-68) years; $51.9 \%$ $(n=42)$ were female and $48.1 \%(n=39)$ were men. Left nephrectomy was performed in $71 \%(\mathrm{n}=64)$ and right nephrectomy in 29\% $(\mathrm{n}=17)$ donors (Table 1$)$.

Intraoperative accessory renal artery was observed in $22.2 \%(\mathrm{n}: 18)$ of the donors; single renal artery in $77.8 \%(n=63)$, double renal artery in $19.8 \%(n=16)$, and triple renal artery in $2.4 \%(n=2)$. Specificity, sensitivity, and accuracy for accessory artery detection in CTA for observer 1 and observer 2 were $88.9 \%, 100 \%$, and $97.5 \%$, respectively (16/18). Excellent agreement among observers was detected (kappa: 1.00; p< 0.001) (Table 2). More than three renal arteries were not detected in any donor. Both observers evaluated the presence of triple renal artery as bilateral renal artery in two donors. Retrospectively, these arteries were identified as superior and inferior polar arteries with a diameter of less than $2 \mathrm{~mm}$, respectively. These arteries were not considered important by the surgeon.
Early bifurcation was detected in $21 \%(n=17)$ of the donors. Specificity, sensitivity, and accuracy for early bifurcation detection in CTA for observer 1 and observer 2 were $98.4 \%, 94.1 \%$, and $97.5 \%$, respectively $(16 / 17)$. High agreement among observers was detected (kappa: 0.853; p< 0.001) (Table 3). The mean length from aorta to renal artery branching was $21.2 \mathrm{~mm}$ in the two donors in which early branching was misregistered, possibly confused with early branching.

Renal vein anomaly was observed in $12.3 \% \quad(n=10)$ of the donors, and all of them were in the left kidney. Specificity, sensitivity, and accuracy for detecting renal vein variation in CTA for observer 1 and observer 2 were 100. Excellent agreement was found between observers (kappa: 1,000; $<<0.001)$. Of the donors with renal vein anomalies, $80 \%(\mathrm{n}: 8)$ had a retroaortic and 20\% (n:2) had a circumaortic renal vein. All of the variations were detected by both observers.

A normal single ureter was detected in 78 of 81 intraoperative kidneys. Variations of the pelvicalyceal system and ureter were observed in $3.7 \%(n=3)$ of the donors. There was one partial ureteral duplication in the right kidney and two complete ureteral duplications in the left kidney. Specificity, sensitivity, and accuracy for detecting renal pelvis and ureter variations on CT for observer 1 and observer 2 were 100\% (3/3). Excellent agreement was found between observers (kappa: 1,00; $\mathrm{p}<0.001)$.

\begin{tabular}{|lc|}
\hline \multicolumn{2}{|c|}{ Table 1. Demographic characteristics of the renal donors } \\
\hline n (\%)/Mean \pm SD \\
\hline Sex \\
$\quad$ Female \\
$\quad$ Male & $42(51.9)$ \\
Age (years) & $39(48.1)$ \\
Side & $49 \pm 12$ \\
$\quad$ Left & \\
Right & $64(79)$ \\
\hline
\end{tabular}

\begin{tabular}{|cccccc|}
\hline \multicolumn{6}{|c|}{ Table 2. Sensitivity, specificity, and accuracy of multidetector CT } \\
angiography for presence of accessory arteries \\
\begin{tabular}{|ccccccc|}
\hline \\
Sensitivite \\
$\%$
\end{tabular} & $\begin{array}{c}\text { Spesifite } \\
\%\end{array}$ & $\begin{array}{c}\text { Accuracy } \\
\text { (\%) }\end{array}$ & Kappa & P \\
\hline Observer 1 & $\begin{array}{c}88.9 \\
(16 / 18)\end{array}$ & $\begin{array}{c}100 \\
(63 / 63)\end{array}$ & 97.5 & 1 & $<0.001$ \\
Observer 2 & $\begin{array}{c}88.9 \\
(16 / 18)\end{array}$ & $\begin{array}{c}100 \\
(63 / 63)\end{array}$ & 97.5 & 1 & $<0.001$ \\
\hline
\end{tabular}

Table 3. Sensitivity. specificity. and accuracy of multidetector CT angiography for presence of early branching of renal arteries

\begin{tabular}{|cccccc|}
\hline & $\begin{array}{c}\text { Sensitivite } \\
\%\end{array}$ & $\begin{array}{c}\text { Spesifite } \\
\%\end{array}$ & $\begin{array}{c}\text { Accuracy } \\
(\%)\end{array}$ & Kappa & P \\
\hline Observer 1 & $\begin{array}{c}94.1 \\
(16 / 17)\end{array}$ & $\begin{array}{c}98.4 \\
(63 / 64)\end{array}$ & 97.5 & 0.853 & $<0.001$ \\
Observer 2 & $\begin{array}{c}94.1 \\
(16 / 17)\end{array}$ & $\begin{array}{c}98.4 \\
(63 / 64)\end{array}$ & 97.5 & 0.853 & $<0.001$ \\
\hline
\end{tabular}




\section{DISCUSSION}

Kidney transplantation remains the most effective treatment option for patients with end-stage renal disease. Transplantation can be done from cadavers or from living kidney donors. According to recent data, living donor transplants account for more than two-thirds of all kidney transplants performed in Turkey, compared to only about a quarter in the United States $(17,18)$.

Donor safety is the primary goal of living donor transplant programs. All potential donors should have standard laboratory tests, clinical evaluation prior to kidney transplant surgery, and anatomical evaluation of the kidney and renal vessels should include imaging studies $(19,20)$.

The number, length, location and branching pattern of the renal arteries, venous anomaly, kidney and collecting system should be evaluated preoperatively by imaging methods (21).

Multi-detector CT angiography (MDCTA), contrastenhanced MR angiography and digital subtraction angiography (DSA) can be applied in the structural evaluation of the kidney and renal vascular system in living kidney donors. However, the use of DSA has decreased because it is invasive and does not provide detailed information about venous anatomy and renal parenchyma. MR imaging is not sensitive in detecting urolithiasis and its spatial resolution is lower than MDCTA $(10,22)$.

MDCT is a non-invasive, easily accessible and relatively inexpensive imaging modality. In addition, MDCT is preferred to other imaging modalities due to its high spatial resolution, acquisition rate, ability to detect small accessory arteries, and more sensitive to identify and characterize vascular calcifications (23).

Adequate vascular augmentation is essential to obtain a high-quality angiogram. The degree of arterial opacification defines the technical proficiency of a CTA scan. It is proportional to the rate and dose of contrast agent administration (24).

The organ taken in donor surgeries is used in another individual and the donor continues his life with the remaining kidney. For this reason, it is of great importance to remove the organ to be transplanted in a way that causes the least damage to the donor and the organ.

CI-AKI is related to the amount of contrast material used, and although it is usually self-limiting, it can lead to increased morbidity and mortality (11). Groups of patients with hypertension, advanced age, and recent exposure to nephrotoxic drugs are at risk of developing CI-AKI. Therefore, the contrast dose should be kept to a minimum, provided it does not affect the overall quality of the image (12).
Recent advances in CT technology have allowed faster image acquisition times, reducing the time required to opacify the pulmonary vasculature. This made it possible to consider reducing the dose of contrast agent used in other angiographies as well as in CT pulmonary angiographies (13).

To the best of our knowledge, our study is the first study in which the diagnostic performance of CTA obtained using low-dose contrast material was compared with surgical results in renal donors. It is aimed to reduce the risk of complications and the cost of screening by reducing the dose, and to increase patient safety.

Although the contrast agent doses used in CTA imaging for kidney donors vary, they have decreased over the years with the development of CT technology. Rankin et al. (14) used $150 \mathrm{ml}$, Kawamoto et al. (25) used $120 \mathrm{ml}$, Ghonge et al. (26) used $100 \mathrm{ml}$ of contrast agent for renal donors.

In our study, CTA had a diagnostic accuracy of $97.5 \%$ for number of arteries when compared with intraoperative findings. Similarly, Sarier et al. (2) reported an accuracy rate of $97.9 \%$ with CTA in their study on 2,144 living donors using an average of $90 \mathrm{ml}$ of contrast. In our study, the specificity and sensitive values for accessory renal artery in CTA were $100 \%$ and $88.9 \%$, respectively, and Sarıer et al. similar to his work.

When CTA and intraoperative findings were compared in terms of early bifurcation in our study, the specificity, sensitivity, and accuracy were $98.4 \%, 94.1 \%$, and $97.5 \%$, respectively. Kawamoto et al. (14) reported that early branching of the renal arteries could be detected with an average accuracy of $96 \%$ in his study by applying $100 \mathrm{ml}$ of contrast material.

Çınar et al. (27) found the retroartic and circumaortic renal vein variation rate of $9.4 \%$ in their study on 504 living donors using 80-100 $\mathrm{ml}$ contrast. Holden et al. (28) found a $14 \%$ vein variation rate in 80 living donors. In the study, this rate was similar to that of Holden et al. We think that this is due to the fact that the number of patients in the study is less than that of Çinar et al., and that it is close to that of Holden et al. In the study, the specificity, sensitivity and accuracy in detecting renal vein variations in MDCT were similar to the study of Turkvatan et al. (15).

The rate of detection of pelvicalyceal system and ureters variation in our study was similar to the literature. The specificity and sensitivity of detection with MDCT was $100 \%$, and it was similar to the study of Türkvatan et al. (15).

In the literature, some authors recommend scanning before contrast application to detect the presence of nephrolithiasis and urolithiasis, while others do not 
$(15,28,29)$. In our study, unhanced imaging was not performed in order to minimize the radiation dose received.

We experienced that nephro and urolithiasis can be detected in arterial phase images. In a literature study of 65 patients using MDCT, all five patients with urolithiasis were seen on both non-contrast and contrast-enhanced scans (30). In our study, we detected four donors, two with nephrolithiasis and two with urolithiasis, on arterial phase images.

The limitations of our study are that it is retrospective, single-centered, and the images were evaluated by only two observers. In addition, renal function tests and the incidence of CI-AKI were not compared with CTA with high-dose contrast material.

\section{CONCLUSION}

Our study showed that CTA performed with low-dose contrast agent $(60 \mathrm{~mL})$ on 128 -slice CT in renal donors is clinically applicable without adversely affecting the image quality and diagnostic value, and provides sufficient preoperative information. New studies are needed to determine that CT with less slices provides sufficient preoperative information.

\section{ETHICAL DECLARATIONS}

Ethics Committee Approval: The study was reviewed and applied by Non-Interventional Clinical Research Ethics Committee of Health Sciences University, Haydarpaşa Numune Training and Research Hospital Health Application Center (Date: 02.08.2021, Decision No: 2021/197-3396).

Informed Consent: All patients signed the free and informed consent form.

Referee Evaluation Process: Externally peer-reviewed.

Conflict of Interest Statement: The authors have no conflicts of interest to declare.

Financial Disclosure: The authors declared that this study has received no financial support.

Author Contributions: All of the authors declare that they have all participated in the design, execution, and analysis of the paper and that they have approved the final version.

\section{REFERENCES}

1. Sarier M, Sepin Ozen N, Guler H, et al. Prevalence of sexually transmitted diseases in asymptomatic renal transplant recipients. Exp Clin Transplant 2018; 1: 1-5.

2. Sarier M, Callioglu M, Yuksel Y, Duman E, Emek M, Usta SS. Evaluation of the renal arteries of 2,144 living kidney donors using computed tomography angiography and comparison with intraoperative findings. Urologia Internationalis 2020; 104: 63740 .
3. Patil UD, Ragavan A, Nadaraj, et al. Helical CT angiography in evaluation of live kidney donors. Nephrol Dial Transplant 2001; 16: $1900-4$.

4. Drager LF, Bortolotto LA, Figueiredo AC, Krieger EM, Lorenzi GF. Effects of continuous positive airway pressure on early signs of atherosclerosis in obstructive sleep apnea. Am J Respir Crit CareMed 2007; 176: 706-12.

5. Raman SS, Pojchamarnwiputh S, Muangsomboon K, et al. Utility of 16-MDCT angiography for comprehensive preoperative vascular evaluation of laparoscopic renal donors. Am J Roentgenol 2006; 186: $1630-8$.

6. Carter JT, Freise CE, McTaggart RA, et al. Laparoscopic procurement of kidneys with multiple renal arteries is associated with increased ureteral complications in the recipient. Am J Transplant 2005; 5: 1312-8.

7. Smith PA, Ratner LE, Lynch FC, Corl FM, Fishman EK. Role of CT angiography in the preoperative evaluation for laparoscopic nephrectomy. Radiographics 1998; 18: 589-601.

8. Liefeldt L, Klüner C, Glander P, et al. Non-invasive imaging of living kidney donors: intraindividual comparison of multislice computed tomography angiography with magnetic resonance angiography. Clin Transplant 2012; 26: E412-7.

9. Chai JW, Lee W, Yin YH, et al. CT angiography for living kidney donors: accuracy, cause of misinterpretation and prevalence of variation. Korean J Radiol 2008; 9: 333-9.

10.Sebastia C, Peri L, Salvador R, et al. Multidetector CT of living renal donors: lessons learned from surgeons. Radiographics 2010; 30: $1875-90$

11.Thomsen HS, Morcos SK, Barrett BJ. Contrast-induced nephropathy: the wheel has turned 360 degrees. Acta Radiol 2008; 49: 646-57.

12.Lewington A, MacTier R, Hoefield R, Sutton A, Smith D, Downes M. Prevention of contrast induced acute kidney injury (CI-AKI) in adult patients. Royal College of Radiologist Guidelines 2013.

13. Bae KT, Tao C, Gürel S, et al. Effect of patient weight and scanning duration on contrast enhancement during pulmonary multidetector CT angiography. Radiology 2007; 242: 582-9.

14. Kawamoto S, Montgomery RA, Lawler LP, Horton KM, Fishman EK. Multi-detector CT angiography for preoperative evaluation of living laparoscopic kidney donors. AJR Am J Roentgenol 2003; 180: $1633-8$

15. Türkvatan A, Akıncı S, Yıldız Ş, Ölçer T, Cumhur T. Multidetector computed tomography for preoperative evaluation of vascular anatomy in living renal donors. Surg Radiol Anat 2009; 31: $227-$ 35 .

16. Han WK, Na JC, Park SY. Low-dose CT angiography using ASiR-V for potential living renal donors: a prospective analysis of image quality and diagnostic accuracy. European Radiology 2019; 30: 798-805.

17. Diniz G, Tugmen C, Sert İ. Türkiye ve Dünyada organ transplantasyonu. Tepecik Eğit ve Araşt Hast Dergisi 2019; 29: 1-10.

18. Black CK, Termanini KM, Aguirre O, Hawksworth JS, Sosin M. Solid organ transplantation in the 21st century. Ann Transl Med 2018; 6: 409-10.

19. Hainninen EL, Denecke T, Stelter L, et al. Preoperative evaluation of living kidney donors using multirow detector computed tomography: comparison with digital substraction angiography and intraoperative findings. Transpl Int 2005; 18: 1134-41.

20.Ethics Committee of the Transplantation Society. The consensus statement of the Amsterdam Forum on the care of the live kidney donor. Transplantation 2004; 78: 491-2.

21.Derauf B, Goldberg ME. Angiographic assessment of potential renal transplant donors. Radiol Clin North Am 1987; 25: 261-5.

22.Jha RC, Korangy SJ, Ascher SM, Takahama J, Kuo PC, Johnson LB. MR angiography and preoperative evaluation for laparoscopic donor nephrectomy. AJR Am J Roentgenol 2002; 178: 1489-95. 
23. Halpern EJ, Mitchell DG, Wechsler RJ, Outwater EK, Moritz MJ, Wilson GA. Preoperative evaluation of living renal donors: comparison of CT angiography and MR angiography. Radiology 2000; 216: 434-9.

24. Fleischmann D. How to design injection protocols for multiple detector-row CT angiography (MDCTA) Eur Radiol 2005;15 Suppl 5: e60-5.

25. Rankin SC, Jan W, Koffman CG. Noninvasive Imaging of Living Related Kidney Donors. Am J Roentgenol 2001; 177: 349-55.

26. Ghonge N, Gadanayak S, Rajakumari V. MDCT evaluation of potential living renal donor, prior to laparoscopic donor nephrectomy: What the transplant surgeon wants to know? Indian J Radiol Imaging 2014; 24: 367.

27. Çınar C, Türkvatan A. Prevalence of renal vascular variations: Evaluation with MDCT angiography. Diagnostic and Interventional Imaging 2016; 97: 891-7.

28. Holden A, Smith A, Dukes P, Pilmore H, Yasutomi M. Assessment of 100 live potential renal donors for laparoscopic nephrectomy with multi-detector row helical CT. Radiology 2005; 237: 973-80.

29. Kim JK, Park SY, Kim H, et al. Living donor kidneys: usefulness of multi-detector row CT for comprehensive evaluation. Radiology 2003; 229: 869-76.

30. Caoili EM, Cohan RH, Korobkin M, et al. Urinary tract abnormalities: initial experience with multi-detector row CT urography. Radiology 2002; 222: 353-60. 